\title{
The utility of urine-circulating miRNAs for detection of prostate cancer
}

\author{
Kristina Stuopelyte ${ }^{1}$, Kristina Daniunaite ${ }^{1}$, Arnas Bakavicius ${ }^{2}$, Juozas R Lazutka ${ }^{1}$, Feliksas Jankevicius ${ }^{2,3}$ and \\ Sonata Jarmalaite ${ }^{\star, 1,3}$ \\ ${ }^{1}$ Division of Human Genome Research Centre, Faculty of Natural Sciences, Vilnius University, Sauletekio Avenue 7, Vilnius \\ LT-10222, Lithuania; ${ }^{2}$ Urology Centre, Vilnius University, Santariskiu 2, Vilnius LT-08661, Lithuania and ${ }^{3}$ National Cancer Institute, \\ Santariskiu 1, Vilnius LT-08406, Lithuania
}

Background: In this paper, the utility of urine-circulating microRNAs (miRNAs) as the potential biomarker of prostate cancer (PCa), the second most prevalent male cancer worldwide, was evaluated.

Methods: Cancerous $(N=56)$ and non-cancerous $(N=16)$ prostate tissues were analysed on TaqMan Low Density Array, with the initial screening of 754 miRNAs in a subset of the samples. The abundance of selected miRNAs was analysed in urine specimens from two independent cohorts of patients with PCa ( $N=215$ overall), benign prostatic hyperplasia (BPH; $N=23)$, and asymptomatic controls ( $A S C ; N=62$ ) by means of quantitative reverse transcription PCR.

Results: Over 100 miRNAs were found deregulated in PCa as compared with non-cancerous prostate tissue. After thorough validation, four miRNAs were selected for the analysis in urine specimens. The abundance of miR-148a and miR-375 in urine was identified as specific biomarkers of PCa in both cohorts. Combined analysis of urine-circulating miR-148a and miR-375 was highly sensitive and specific for PCa in both cohorts ( $\mathrm{AUC}=0.79$ and 0.84 ) and strongly improved the diagnostic power of the PSA test $(A \cup C=0.85$, cohort PCa1), including the grey diagnostic zone $(A \cup C=0.90)$.

Conclusions: Quantitative measurement of urine-circulating miR-148a and miR-375 can serve as the non-invasive tool for sensitive and specific detection of PCa.

Prostate cancer $(\mathrm{PCa})$ is the second most prevalent oncological disease and the fifth leading cause of cancer-related death among men worldwide. PCa-related mortality rates are extremely high in the Nordic and Baltic European countries, including Lithuania (Bray et al, 2010). The primary tool for PCa diagnosis is the widely used prostate-specific antigen (PSA) test. However, there is a number of important limitations of this popular serum marker, including the modest ability to accurately distinguish patients with and without $\mathrm{PCa}$ and those who harbour an aggressive form of the disease (Duffy, 2014). To overcome these limitations, there is a need to replace or at least to refine the diagnostic accuracy of PSA testing with novel PCa-specific biomarkers. Nowadays, dozens of new molecular biomarkers have been suggested for PCa diagnostics (Velonas et al, 2013; Ziaran et al, 2015), but none of them are confirmed and proceeded to clinical use so far. High sensitivity, specificity, and precision are prerequisite for modern biomarkers. Novel diagnostic tools should also meet some practical issues, such as easy accessibility of test material, simplicity and repeatability of procedure, low cost, and, of course, minimal discomfort to patients.

MicroRNAs (miRNAs) are about 22 nucleotides long noncoding RNAs that regulate gene expression posttranscriptionally by either repressing or degrading mRNA transcripts. MiRNAs are involved in almost all biological processes, including cell proliferation, development, differentiation, and metabolism. Deregulated expression of miRNAs promotes development of various diseases, including cancer (Xu et al, 2014). Expression profiles of miRNAs obtained from malignant and benign tissues differ significantly. As tumour cells can release miRNAs into circulation, the abundance of particular miRNAs in bodily fluids

*Correspondence: Professor S Jarmalaite; E-mail: sonata.jarmalaite@gf.vu.lt

Received 19 April 2016; revised 11 July 2016; accepted 12 July 2016; published online 4 August 2016

(c) 2016 Cancer Research UK. All rights reserved 0007-0920/16 
might reflect the events in tumorigenesis and serve as the noninvasive diagnostic or even prognostic tool (Taylor and GercelTaylor, 2008). Circulating miRNAs are quite stable in biological fluids, as RNA-binding proteins or microvesicles protect them from most RNA-degrading agents (Cortez et al, 2011) and, therefore, are suitable for diagnostic purposes in routine studies (Sita-Lumsden et al, 2013). In recent years, several PCa-related circulating miRNAs have been identified and proposed as a tool for early and specific detection of the disease (Sapre and Selth, 2013; Sita-Lumsden et al, 2013; Jackson et al, 2014). The vast majority of PCa-specific miRNAs were studied in blood (Kelly et al, 2013; Jackson et al, 2014), where miRNAs excreted from various organs can be detected. Meanwhile, more relevant fluids, such as urine, seminal fluid, or ejaculate, remained less well tested for the presence of PCa-specific miRNAs (Dijkstra et al, 2014; Mlcochova et al, 2014).

For selection of PCa-specific miRNAs with diagnostic potential, expression levels of several hundreds of miRNAs were analysed in cancerous and non-cancerous prostate tissues on TaqMan Low Density Array (TLDA) cards. A set of PCa-specific miRNAs was further quantified in the urine of PCa cases from two independent cohorts and controls. Subsequently, the diagnostic power of selected urinary miRNAs as the independent diagnostic biomarkers was established and the possibility to improve the accuracy of the PSA test in detecting PCa was assessed.

\section{MATERIALS AND METHODS}

Tumour and urine sample collection. Specimens (tissue and/or urine) were collected prospectively from 237 PSA-screened and biopsy-proven $\mathrm{PCa}$ patients who subsequently underwent radical prostatectomy (RP) at the Urology Department of Vilnius University Hospital Santariskiu Clinics from January 2008 to January 2014. For comparison, urine specimens from 23 benign prostatic hyperplasia $(\mathrm{BPH})$ patients and 62 asymptomatic control (ASC) men with PSA level $<3 \mathrm{ng} \mathrm{ml}^{-1}$ were collected. The study was approved by the Lithuanian Bioethics Committee, and all patients gave informed consent for participation. The research was a part of a large-scale PCa biomarker study started at 2008 and conducted according to standardised protocols of sample collection and processing reported previously (Sabaliauskaite et al, 2012; Daniunaite et al, 2014). Cancerous ( $\geqslant 70 \%$ of tumour cells) and non-cancerous $(0 \%)$ prostatectomy tissues were sampled by expert histopathologist as previously reported (Sabaliauskaite et al, 2012; Daniunaite et al, 2014) and prepared for molecular analysis.

For miRNA detection in prostate tissues, fresh-frozen tissue sections from RPs of 72 PCa patients were available, including $56 \mathrm{PCa}$ and 16 non-cancerous prostate tissues (NPTs). A set of these specimens (42 PCa and $12 \mathrm{NPTs}$ ) was included in global miRNA expression profiling on human miRNA TaqMan arrays, while an extended number of prostate tissue samples (52 PCa and 12 NPTs) was used for thorough analysis of selected miRNAs on custom-designed TLDA cards (Figure 1).

Based on final pathology, PCa specimens were defined as pathological tumour stage $2(\mathrm{pT} 2 ; N=38)$ or pT3 $(N=18)$ tumours (Table 1). Gleason score $(\mathrm{G})$ was obtained according to International Society of Urological Pathology 2005 recommendations (Epstein et al, 2005), and 20 patients had tumours of G6, while others of G7 or G8/9 $(N=36)$. G7 tumours were further substratified according to the sum of primary and secondary tumour grades $(3+4$ and $4+3)$, and $G \leqslant(3+4)$ and $G \geqslant(4+3)$ groups were composed of 49 and 7 cases, respectively. Positive surgical margins were obtained in 22 patients, while 34 patients were negative for surgical margin status. Follow-up data were collected for all but $1 \mathrm{PCa}$ case (55 out of 56, 98.2\%), and the mean

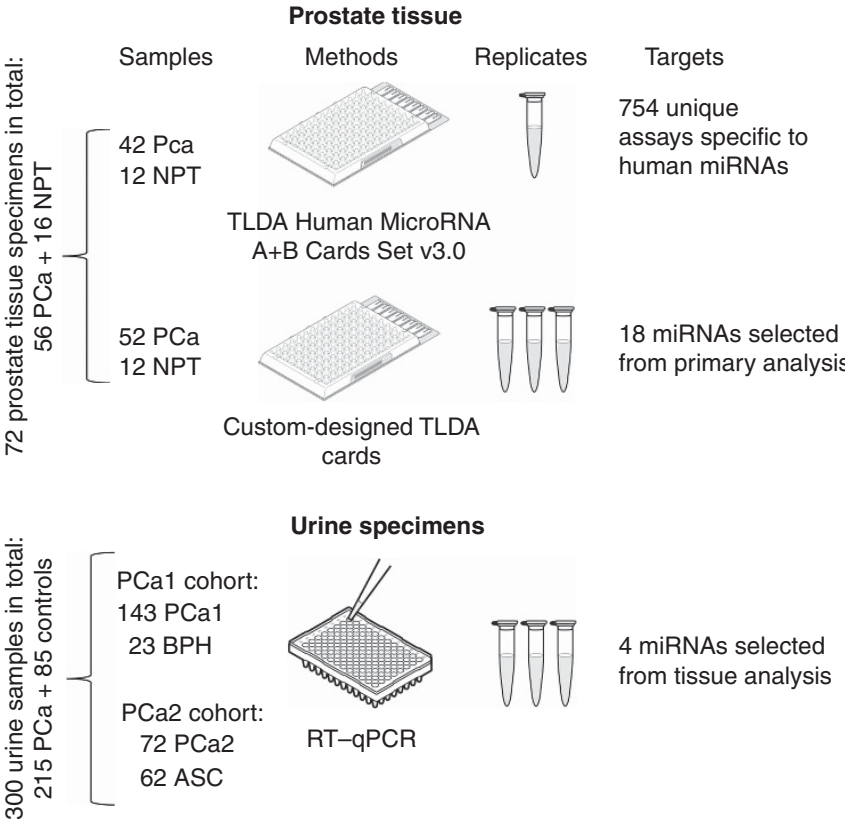

Figure 1. Workflow of miRNA expression study in tissue and urine of PCa patients and controls.

follow-up time was 2.8 years. Eighteen out of the 55 (32.7\%) PCa patients experienced biochemical recurrence (BCR) after RP. BCR following RP was defined by two consecutive PSA values of $\geqslant 0.2 \mathrm{ng} \mathrm{ml}^{-1}$ and rising. The fusion transcript TMPRSS2-ERG status of the tumours was known from our previous studies (Sabaliauskaite et al, 2012; Demidenko et al, 2015).

For circulating miRNA extraction, urine from two independent cohorts was used (Figure 1). Preoperative catheterised urine was available from the first cohort (PCa1) and BPH cases, while initial voided urine was collected before surgery from the second cohort (PCa2) and ASC cases. PCa1 group consisted of 143 PCa patients, with a set of the cases $(N=42)$ included in aforesaid prostate tissue study. The mean follow-up period of PCa1 cohort was 2.7 years, and the follow-up data were collected for $91.6 \%$ (131 out of 143) of the cases with urine analysed. PCa2 group consisted of $72 \mathrm{PCa}$ patients who had the shorter mean follow-up period (1.0 year), and follow-up data were missing for a set (18 out of 72) of the cases. ASC cases were age-matched (mean 63.3 years) to both the PCa groups. Detailed data of all the study groups are provided in Table 1.

Urine samples from both PCa cohorts and controls were processed using the same protocol: $30 \mathrm{ml}$ of urine was centrifuged at 1000 r.p.m. for $15 \mathrm{~min}$ at $4{ }^{\circ} \mathrm{C}$ and up to $1.5 \mathrm{ml}$ of lower fraction was collected. PBS-washed and suspended urine specimens were stored at $-70{ }^{\circ} \mathrm{C}$ until use.

RNA extraction. Total RNA from prostate tissue was isolated with the mirVana miRNA Isolation Kit (Ambion, Austin, TX, USA; Thermo Fisher Scientific, Waltham, MA, USA) according to the manufacturer's protocol. Approximately $30 \mathrm{mg}$ of snap-frozen and ground tissue were treated with $600 \mu \mathrm{l}$ of Lysis/Binding Buffer and purified RNA was eluted in $100 \mu$ l of Elution Solution.

Total RNA from urine was extracted using the miRNeasy Mini Kit (Qiagen, Valencia, CA, USA) according to the manufacturer's protocol with some modifications. In all, $200 \mu \mathrm{l}$ of urine sediments were mixed with five sample volumes of Qiazol reagent, spiked with $25 \mathrm{fmol}$ synthetic cel-miR-39 (Qiagen), and one sample volume of chloroform was added. From that step, the manufacturer's protocol was followed, and finally, samples were eluted twice in $30 \mu \mathrm{l}$ of RNase-free water. 
Table 1. Clinical-pathological characteristics of patients with prostate cancer (PCa), benign prostatic hyperplasia (BPH), and asymptomatic controls (ASCs)

\begin{tabular}{|c|c|c|c|c|c|c|}
\hline & \multicolumn{2}{|c|}{ Tissue $(N=72)$} & \multicolumn{4}{|c|}{ Urine $(N=300)$} \\
\hline \multicolumn{7}{|l|}{ Age } \\
\hline \multicolumn{7}{|l|}{ Gleason score, G } \\
\hline $\begin{array}{l}\text { 6; n (\%) } \\
\geqslant 7 ; n(\%) \\
\text { Unknown; n (\%) }\end{array}$ & $\begin{array}{c}20(35.7) \\
36(64.3) \\
-\end{array}$ & - & $\begin{array}{c}75(52.4) \\
68(47.6) \\
-\end{array}$ & $\begin{array}{c}15(20.8) \\
56(77.8) \\
1(1.4)\end{array}$ & - & - \\
\hline \multicolumn{7}{|c|}{ Sub-stratified Gleason score, G } \\
\hline $\begin{array}{l}\leqslant(3+4) ; n(\%) \\
\geqslant(4+3) ; n(\%) \\
\text { Unknown; } n(\%)\end{array}$ & $\begin{array}{c}49(87.5) \\
7(12.5) \\
-\end{array}$ & - & $\begin{array}{l}118(82.5) \\
10(7.0) \\
15(10.5)\end{array}$ & $\begin{array}{c}53(73.6) \\
18(25.0) \\
1(1.4)\end{array}$ & - & - \\
\hline \multicolumn{7}{|c|}{ Pathological stage, pT } \\
\hline $\begin{array}{l}\text { Yes; n (\%) } \\
\text { No; } n(\%) \\
\text { Unknown; n (\%) }\end{array}$ & $\begin{array}{c}18(32.1) \\
37(66.1) \\
1(1.8)\end{array}$ & - & $\begin{array}{r}26(18.2) \\
105(73.4) \\
12(8.4)\end{array}$ & $\begin{array}{r}9(12.5) \\
45(62.5) \\
18(25.0)\end{array}$ & - & - \\
\hline \multicolumn{7}{|l|}{ Serum PSA } \\
\hline 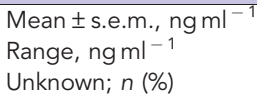 & $\begin{array}{c}10.8 \pm 4.5 \\
2.8-84.2 \\
2(3.6)\end{array}$ & $\begin{array}{c}6.6 \pm 1.8 \\
2.6-17.0 \\
-\end{array}$ & $\begin{array}{c}9.7 \pm 1.0 \\
0.1-84.2 \\
3(2.1)\end{array}$ & $\begin{array}{c}8.5 \pm 0.9 \\
2.3-38.0 \\
-\end{array}$ & $\begin{array}{c}7.8 \pm 1.3 \\
0.8-28.1 \\
2(8.7)\end{array}$ & - \\
\hline \multicolumn{7}{|l|}{ TMPRSS2-ERG } \\
\hline $\begin{array}{l}\text { Yes; n (\%) } \\
\text { No; } n(\%) \\
\text { Unknown; n (\%) }\end{array}$ & $\begin{array}{c}37(66.1) \\
19(33.9) \\
-\end{array}$ & - & $\begin{array}{l}56(39.2) \\
41(28.7) \\
46(32.2)\end{array}$ & $\begin{array}{l}16(22.2) \\
10(13.9) \\
46(63.9)\end{array}$ & - & - \\
\hline \multicolumn{7}{|l|}{ Surgical margins } \\
\hline
\end{tabular}

RNA quality was evaluated using NanoDrop 2000 spectrophotometer (Thermo Scientific, Wilmington, DE, USA) and 2100 Bioanalyzer (Agilent Technologies, Santa Clara, CA, USA), and stored at $-70^{\circ} \mathrm{C}$ until use.

TaqMan Low Density arrays. For miRNA profiling, TLDA Human MicroRNA cards (Human MicroRNA A + B TLDA cards Set v.3.0; Applied Biosystems, Carlsbad, CA, USA; Thermo Fisher Scientific) were used, while the custom-designed TLDA cards (Applied Biosystems, Thermo Fisher Scientific) were ordered for further study of expression levels of selected miRNAs in three replicates (Figure 1). For both panels, extracted RNA was reverse transcribed into cDNA using the TaqMan MicroRNA Reverse Transcription Kit (Applied Biosystems, Thermo Fisher Scientific) according to the manufacturer's protocols for cDNA synthesis without preamplification step. Reverse transcription (RT) reaction for $\mathrm{A}+\mathrm{B}$ TLDA was performed in $7.5 \mu \mathrm{l}$ volume and contained Megaplex RT primers (Human Pool Set v3.0) and $500 \mathrm{ng}$ of input RNA, whereas the RT reaction for custom-designed arrays was run in $15 \mu \mathrm{l}$ volume and contained Custom MicroRNA RT pool (Applied Biosystems, Thermo Fisher Scientific) and $350 \mathrm{ng}$ of input RNA. Quantitative PCR (qPCR) was carried out on ViiA 7 Real Time PCR System (Applied Biosystems, Thermo Fisher Scientific).

Quantitative RT PCR (RT-qPCR). In urine specimens (Figure 1), abundance of selected mature miR-148a, -365, -375, and -429 was evaluated by TaqMan Human MiRNA Assays (assay numbers 000470, 001020, 000564, and 001024, respectively,
Applied Biosystems, Thermo Fisher Scientific). RT was performed using the TaqMan MicroRNA Reverse Transcription Kit according to the manufacturer's protocol in $15 \mu \mathrm{l}$ volume, each reaction containing specific stem-loop RT primer and $5 \mu$ l of total RNA from urine. In all, $1.33 \mu \mathrm{l}$ of RT product were further amplified in triplicates in $20 \mu \mathrm{l}$ qPCR reaction volume using TaqMan Universal PCR Master Mix, sequence specific primers, and TaqMan probes. Reactions were performed on ViiA 7 Real Time PCR System (Applied Biosystems, Thermo Fisher Scientific).

RT-qPCR data preprocessing, analytical validation, normalisation, and statistical analysis. For RT-qPCR data analysis, quantification cycle $(\mathrm{Cq})$ values were determined using the ViiA 7 Software v1.2.1 with automatic baseline (Cq 3-15) and threshold selection parameters, except that for the screening step the threshold was manually set to 0.2 . For data preprocessing, expression was considered detected when $\mathrm{Cq}$ values were $<40$ for at least two out of the three replicates. The cutoff percentage of raw $\mathrm{Cq}$ values were determined according to the smallest variable group to avoid the loss of possible associations. The A and B cards were combined according to the expression of U6 nuclear RNA (RNU6) and small nucleolar RNAs (RNU44 and RNU48). Analytical validation of the individual RT-qPCR assays was carried out according to standard procedures, that is, intraassay and interassay variation was checked in three and two replicates, respectively, and the coefficients of variation were calculated. 
For data normalisation, miRNAs with the smallest variation between samples according to NormFinder and geNorm algorithms in TLDA cards were chosen as endogenous controls (ECs), instead of the manufacturer's recommended RNU6, RNU44, and RNU48. Global approach was used as an alternative mean for data normalisation. In urine analysis, variation in RNA isolation efficiency was eliminated by scaling all the values to the spike-in control (cel-miR-39). The values were further converted to relative quantities (NPTs as a reference group for tissues and ASCs for urine) and $\log _{2}$-normalised.

Data preprocessing, filtering, and statistical analysis were performed using GenEx v.6.0.1 (MultiD Analyses AB, Göteborg, Sweden) and STATISTICA v.8.0 (StatSoft, Tulsa, OK, USA) software. Continuous $\log _{2}$-normalised miRNA expression (tissue) and abundance (urine) values were used for all statistical tests. Quantitative variables were compared using unpaired two-tailed $t$-test. Pearson's correlation coefficient was calculated to evaluate correlations. The sensitivity and specificity of diagnostic tests were evaluated using Receiver Operating Characteristic (ROC) curves, and the area under the ROC curve (AUC) was calculated to evaluate how well diagnostic groups are distinguished. To combine biomarkers, logistic regression analysis was applied. Statistically significant differences were set at $P<0.05$.

\section{RESULTS}

MiRNA expression in prostate tissue. Of the 754 human miRNAs provided in the Human MicroRNA A + B TLDA cards, a total of 412 miRNAs met preprocessing criteria and were considered as expressed in prostate tissues. For the data normalisation, six miRNAs showing the smallest variation of expression (let-7g, miR-660, miR-532-3p, miR-30c, miR-103, and miR-26b) were selected from the list of all analysed miRNAs and were used as ECs. In addition, the global data normalisation was performed, and a number of overlapping associations was revealed by using both normalisation methods (Figures 2A and B). Though both methods of data normalisation were used in all steps of the study, only globally normalised data are presented in the text for simplicity.

PCa comparison to NPTs identified a panel of 113 differently $(P<0.05)$ expressed miRNAs, 55 of them were upregulated and 58 were downregulated (Supplementary Table S1). Most significantly deregulated miRNAs $(N=11)$ are depicted in Figure 2C. Other significant associations between the expression levels of miRNAs and clinical variables or TMPRSS2-ERG status are presented in Supplementary Table S1.

Expression levels of those 11 miRNAs showing the most significant deregulation in PCa tissue in comparison to NPTs (Figure 2C) were further analysed in triplicate on custom-designed TLDA cards. In addition, the probes for two miRNAs significantly deregulated in BCR-positive vs BCR-negative cases (miR-365 and miR-505*), and five miRNAs significantly deregulated in TMPRSS2-ERG-positive vs TMPRSS2-ERG-negative cases (let-7b, miR-20a, miR-21, miR-195, and miR-429) were also loaded on the array (Supplementary Table S2).

In this step of the study, where all selected miRNAs were analysed in triplicates in a larger set of samples, four (miR-375,-148a, -31, and -19a) out of the 11 miRNAs showed significantly different expression levels in PCa comparing to NPTs (Figure $3 \mathrm{~A}$ ). As in TLDA screening cards, the expression of miR429 was increased in the fusion gene-positive PCa cases $(P=0.008)$ and the expression level of miR-505* was higher $(P=0.048)$ in BCR-positive cases (Figure 3A). Several significant associations were identified between the expression levels of these miRNAs and pathological stage, Gleason score, surgical margin status, and other clinical variables (Figure 3A and Supplementary Table S2).

MiRNA analysis in urine. MiRNAs abundantly expressed in prostate tissues and showing predominant increase in $\mathrm{PCa}$ in general (miR-375 and miR-148a) or in particular subgroups
A

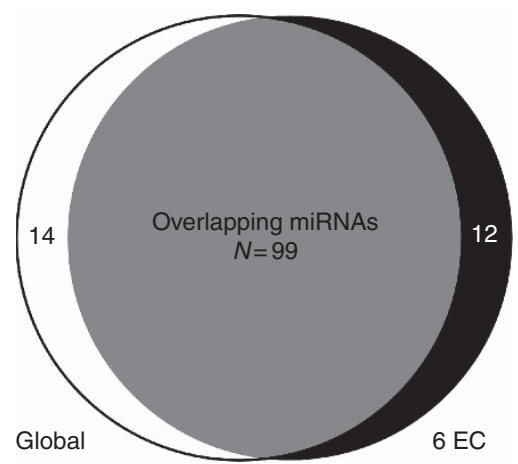

B

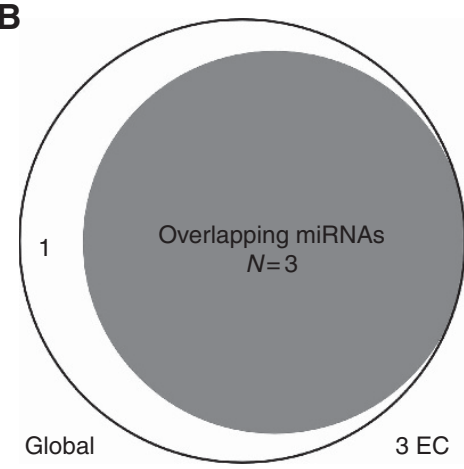

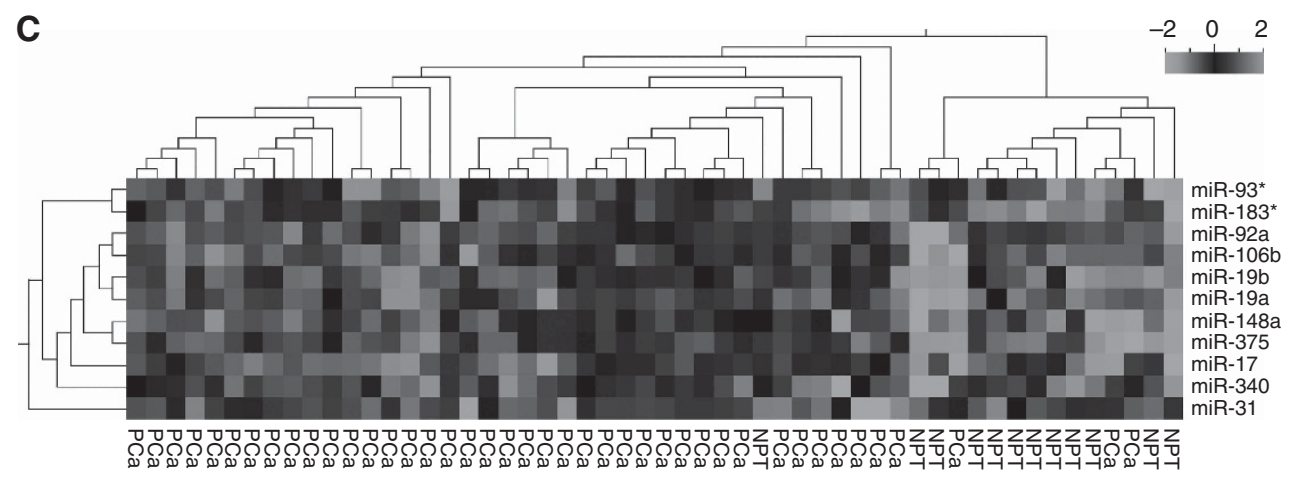

Figure 2. Differentially expressed miRNAs in prostate cancer (PCa) vs NPTs assayed on TLDA Human MicroRNA cards. MiRNA expression was analysed applying global normalisation method (white) or selected ECs (black) in the (A) screening and (B) validation steps. (C) The heat map illustrates the expression of the 11 most significantly deregulated miRNAs in the screening step. A full colour version of this figure is available online. 
A

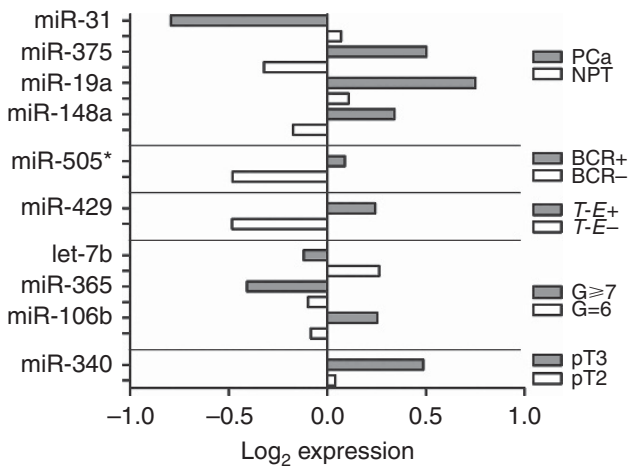

B

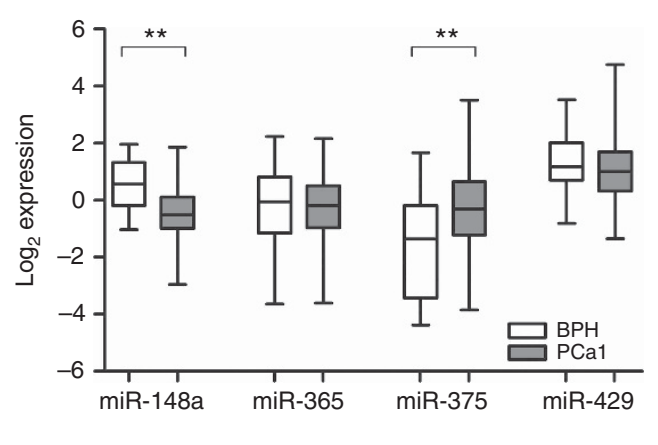

C

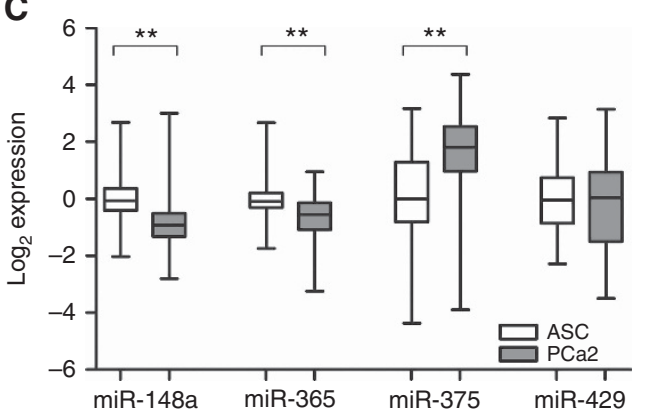

D

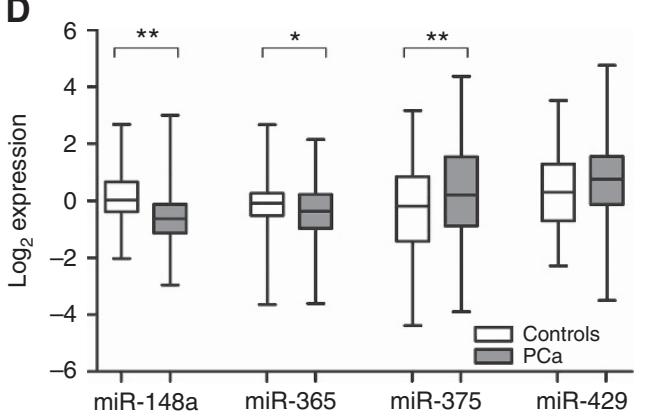

Figure 3. MiRNA levels in prostate tissues and urine specimens. Significant ( $P$-value $<0.05$ ) associations between $(\mathbf{A})$ miRNAs assayed on human microRNA custom-design cards and characteristics of PCa and miRNA levels in urine from PCa cases and controls: (B) PCa1 cohort vs BPH, (C) PCa2 cohort vs ASCs, and (D) all PCa cases vs combined controls. Column bars and lines within boxes indicate the median values of log $2^{-}$ normalised data, whiskers - minimum to maximum, ${ }^{\star} P<0.05$, and ${ }^{*} P<0.001$. Abbreviations: $T$ - $E+/-=T M P R S S 2-E R G$ fusion gene-positive/negative tumours; $\mathrm{G}=$ Gleason score; $\mathrm{PT}=$ pathological stage.

(miR-429 and miR-365) were selected for the analysis in urine specimens. First, the abundance of these miRNAs was analysed in catheterised urine specimens from the PCal cohort (143 PCal and $23 \mathrm{BPH}$ cases). The level of circulating miR-375 was significantly higher $(P<0.001)$, whereas miR-148a lower $(P<0.001)$ in the urine from cases with $\mathrm{PCal}$ in comparison to $\mathrm{BPH}$ (Figure $3 \mathrm{~B}$ ). Besides, the higher level of miR-375 was detected in the urine from patients with pT2 $(P=0.003)$, while miR-429 with pT3 $(P=0.029)$ tumours (Supplementary Table S3).

Next the levels of the same miRNAs were evaluated in the urine of the PCa2 cohort with the voided urine specimens collected preoperatively from $\mathrm{PCa}$ cases $(N=72)$ and compared with the voided urine of healthy controls $(N=62)$. Similarly to PCa1, the same trend in miR-148a and miR-375 abundance was identified in the urine of PCa2 $(P<0.001$; Figure 3C). Furthermore, the levels of urinary miR-365 significantly $(P<0.001)$ differed between PCa2 cases and ASCs. In PCa2, miR-375 was more abundant $(P=0.037)$ in the urine of TMPRSS2-ERG-positive cases (Supplementary Table S3).

In combined analysis of the data from both PCa cohorts and controls, the higher level of circulating miR-375 and the lower level of miR-148a remained independent separators between PCa and control cases (BPHs and ASCs combined; $P<0.001$ ). Besides, miR365 level was also significantly different $(P=0.019)$ in the urine from combined PCa and control cases (Figure 3D). The predominance of miR-375 in the urine of $\mathrm{PCa}$ patients with tumours of lower stage (pT2 vs pT3) also retained statistical significance in this combined analysis (Supplementary Table S3).

Diagnostic value of urinary miRNAs. The sensitivity and specificity to $\mathrm{PCa}$ of the two most promising urinary miRNAs (miR-148a and miR-375) were depicted by ROC curves. Comparisons were made between PCa cohorts and corresponding controls, that is, PCa1 vs $\mathrm{BPH}$ and $\mathrm{PCa} 2$ vs ASC (Figure 4). Estimates of AUC were 0.78 and 0.82 for miR-148a (Figures $4 \mathrm{~A}$ and D) and 0.68 and 0.80 for miR-375 (Figures 4B and E) in PCa1 and PCa2 analyses, respectively. Combined analysis of both urinary miRNAs showed even better diagnostic value in both cohorts (Figures $4 \mathrm{C}$ and F).

Widely used diagnostic PSA test has high rates of false-positive and false-negative results. Using data from the PCa1 cohort, we compared the diagnostic power of urinary miRNAs and serum PSA (Figure 5) and verified the possibility to obtain an improved diagnostic accuracy by combining both tests. All measures and the overall diagnostic power of urinary miR-148a and miR-375 levels to detect PCa were higher than that of the PSA level alone (AUC value $0.78,0.68$, and 0.51 , respectively). Analysis of urinary miRNAs together with the PSA strongly improved the sensitivity ( $84 \%$ vs $64 \%)$ and specificity ( $76 \%$ vs $52 \%)$ and the overall diagnostic power (AUC value 0.85 vs 0.51 ) of this test (Figures 5A and B).

In addition, the diagnostic accuracy of urinary miR-148a and miR-375 and serum PSA was tested in the subgroup of cases falling into the diagnostic grey zone (PSA level $4-10 \mathrm{ng} \mathrm{ml}^{-1}$ ), where the lack of specificity of the PSA test has the most significant clinical consequences. In the PCal cohort, 76 out of the 143 (53.1\%) PCa cases and 11 out of the $23(47.8 \%) \mathrm{BPH}$ cases belonged to the grey zone. In this subgroup, the PSA test was moderately specific (55\%), but the specificity was markedly improved with the combined testing of the two urinary miRNAs (Figures 5C and D). In the PSA grey zone, high sensitivity and specificity values were reached ( $84 \%$ and $82 \%$ ) for PCa by this combined test, while the diagnostic value of the test was close to 0.90 (Figure 5D).

\section{DISCUSSION}

Urine, as a source of tumour-derived circulating nucleic acids, is a valuable disease-specific clinical material for diagnostics of urological malignancies (Payne et al, 2009). However, the profile 
of urine-circulating miRNAs is poorly characterised in PCa. In our study, after screening of miRNA profile in prostate tissues, four $\mathrm{PCa}$-specific and highly expressed miRNAs were selected for the evaluation of their diagnostic potential in urine specimens from PCa cases and controls. Urine-circulating miR-148a and miR-375 showed significant diagnostic potential in two independent cohorts and markedly improved specificity and overall diagnostic power of the PSA test, even in the diagnostic grey zone of PCa.

In our study, miR-375 was significantly overexpressed in PCa tissues and abundant in urine specimens from $\mathrm{PCa}$ patients.
A

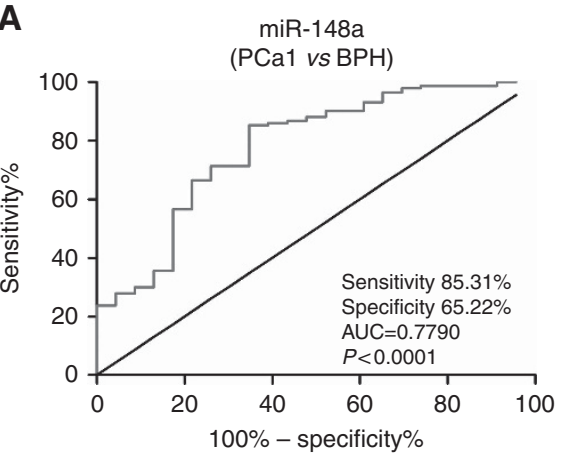

D

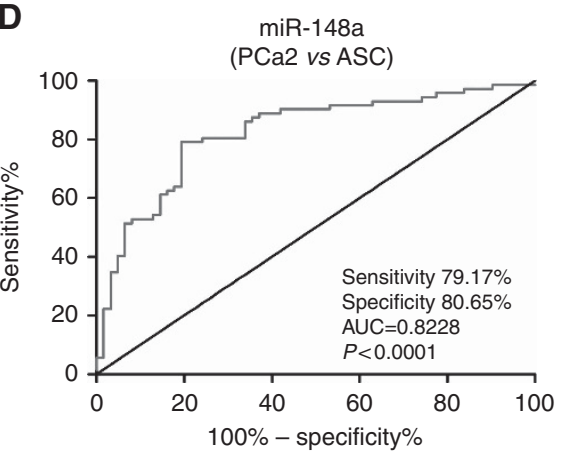

B

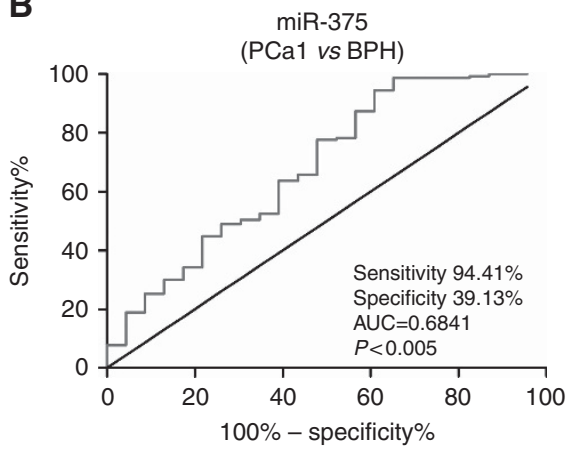

E

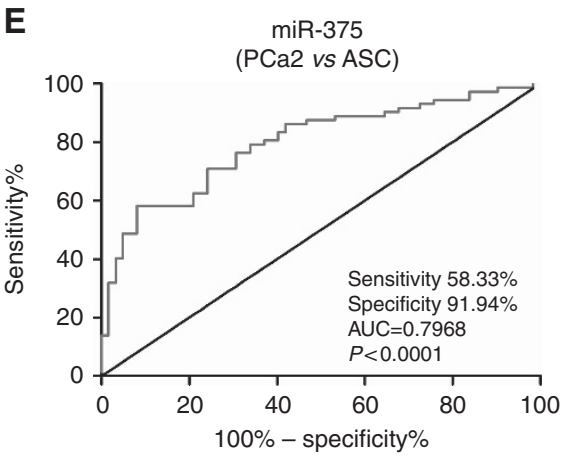

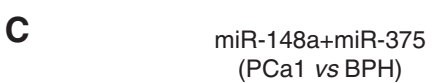

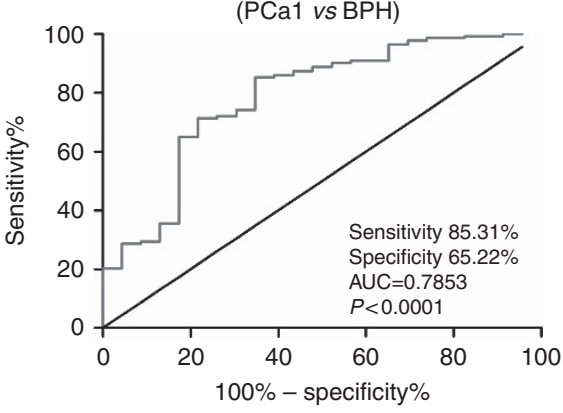

$\mathbf{F}$

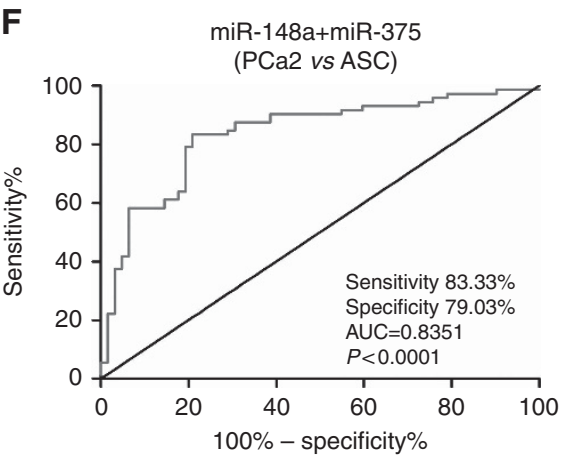

Figure 4. ROC curve analysis of PCa biomarkers. Urinary miR-148a, miR-375, and combination of them in (A-C) cohort PCa1 and (D-F) in cohort PCa2.

A

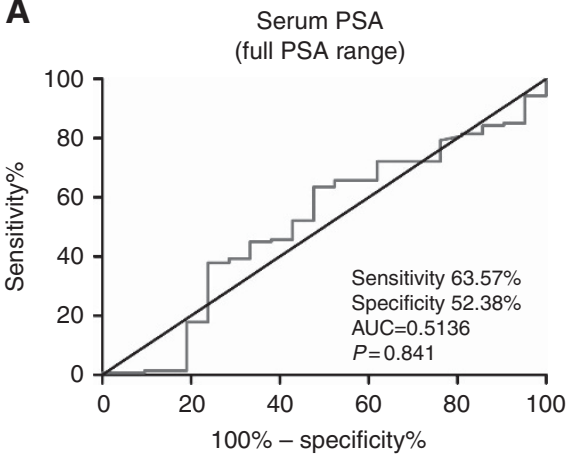

C

Serum PSA

(PSA range 4-10 $\mathrm{ng} \mathrm{ml}^{-1}$ )

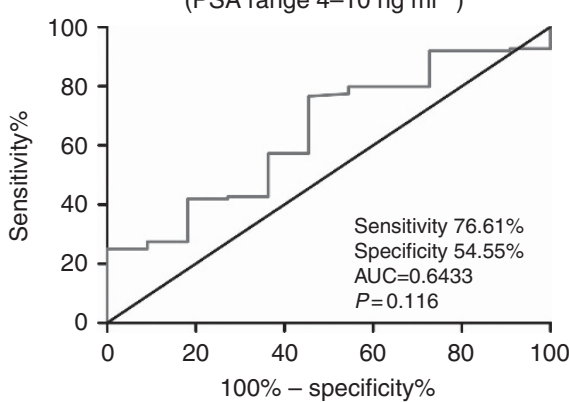

B Serum PSA + miR-148a+miR-375 (full PSA range)

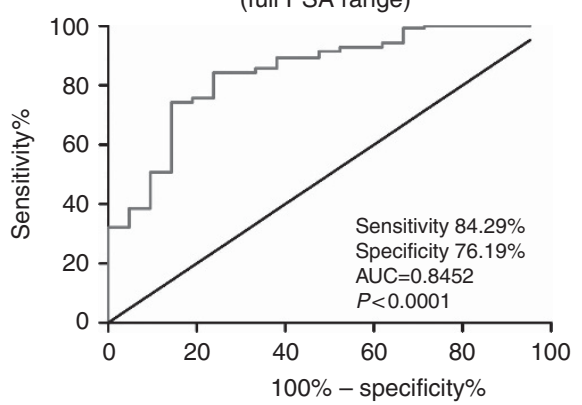

D Serum PSA + miR-148a+miR-375 (PSA range 4-10 $\mathrm{ng} \mathrm{ml}^{-1}$ )

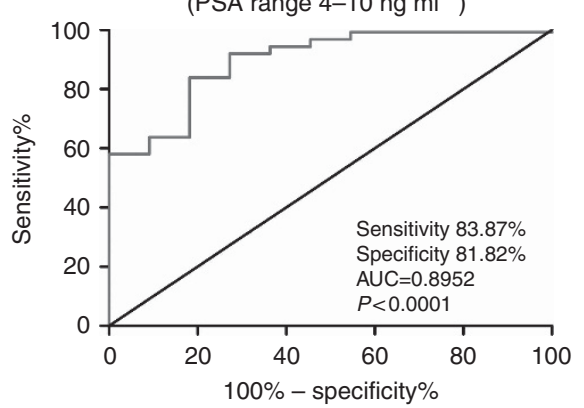

Figure 5. ROC curve analysis of PCa biomarkers and PSA. (A) Serum PSA and (B) combination of urinary miRNAs with PSA in (C) all PSA range and (D) in PSA grey zone (4-10 $\mathrm{ng} \mathrm{ml}^{-1}$ ) only. Analyses were carried out in the PCa1 cohort only. 
Generally, this miRNA is well known as a tumour suppressor and thus is characterised by reduced expression in various tumours; however, its upregulation has been shown in breast and prostate cancer (Brase et al, 2011; Nguyen et al, 2013; Chen et al, 2015). In $\mathrm{PCa}$, increased expression of miR-375 was identified in multiple studies where tumour tissues were compared with NPTs (Ambs et al, 2008; Schaefer et al, 2010; Szczyrba et al, 2010; Wach et al, 2012). Besides, high levels of miR-375 have been shown in plasma and serum from PCa patients in comparison to healthy controls (Brase et al, 2011; Bryant et al, 2012; Selth et al, 2012; Cheng et al, 2013; Nguyen et al, 2013; Huang et al, 2015). Moreover, increased amounts of miR-375 in tissue and blood were associated with aggressive course of the disease. Blood-circulating miR-375 showed significant associations with $\mathrm{PCa}$ progression and metastasis of castration-resistant PCa (CRPC) (Brase et al, 2011; Selth et al, 2012; Cheng et al, 2013; Nguyen et al, 2013). Blood-derived exosomal miR-375 was also predictive for metastatic PCa (Bryant et al, 2012) and showed significant association with overall survival (Huang et al, 2015). Despite extensive miR-375 studies in the blood of PCa patients, urinary miR-375 was analysed only in one study (Bryant et al, 2012). In this study, with 135 urine specimens involved in the analysis, levels of miR-375 did not differ significantly in urine from PCa patients and controls. Contrarily, in our study, the levels of miR-375 were significantly increased in urine specimens of two independent PCa cohorts, especially in the cases with pT2 tumours, emerging as the early-stage PCa biomarker. Therefore, previous studies together with our data suggest PCa-specific origin of circulating miR-375 and reveal its suitability for non-invasive diagnostics.

In PCa, miR-148a is considered as an oncogenic miRNA having an important role in hormone-refractory, drug-resistant PCa and CRPC (Fujita et al, 2010; Jalava et al, 2012). It has been shown that miR-148a promotes cancer cell proliferation (Jalava et al, 2012) and thus contributes to PCa progression (Murata et al, 2010). This miRNA is androgen responsive (Murata et al, 2010) and its overexpression was demonstrated in AR-positive cancer cell lines (Fujita et al, 2010). In our study, upregulation of miR-148a was observed in PCa tissues, whereas in urine reduced rather than increased level of miR-148a was detected in PCa cases from the two cohorts. Similarly, Corcoran et al (2014) found miR-148a to be significantly downregulated in docetaxel-resistant cell lines and exosomes. This expression pattern was also confirmed by the analysis (Corcoran et al, 2014) of publicly available clinical cohorts, where levels of miR-148a were found decreased in urine from PCa vs BPH. In our study, good diagnostic accuracy of this miRNA was demonstrated when analysed alone or in combination with miR375. Additionally, significant improvement in the specificity of the PSA test was reached when both urinary miRNAs were included into the diagnostic panel.

In our study, a combination of two urinary miRNAs showed high sensitivity and specificity for $\mathrm{PCa}$ in both the analysed cohorts. Recent studies suggest that panels of biomarkers outperform diagnostic power of single biomarker such as PSA, thus various combinations of miRNAs have been tested for noninvasive detection of PCa. Similarly to our data, high diagnostic power (AUC value 0.95) was reached when four miRNAs (miR-361-3p, -133b, -221, and -203) from prostatic secretions were used for separation of PCa patients from BPH cases (Guzel et al, 2015). Seminal fluid (Selth et al, 2014) or ejaculate-derived miRNAs (Roberts et al, 2015), including miR-375, showed better diagnostic power than serum PSA. Suitability of urinary miR-1825 and miR-484 was demonstrated for specific detection of $\mathrm{PCa}$ patients among BPH cases (Haj-Ahmad et al, 2014). Very few studies assessed the diagnostic performance of miRNA-based biomarkers within the intermediate PSA range. More recently, Yun et al (2015) revealed that two viral miRNAs circulating in urine of $\mathrm{PCa}$ and BPH patients had higher diagnostic value than PSA, when assessed in the grey zone cohort. Similarly, in our analysis, a small test panel combining PSA with two urinary miRNAs was demonstrated as a powerful tool for PCa detection, even among the cases within the diagnostic grey zone. The sensitivity and specificity of such a combined test in the grey zone was $>80 \%$ with AUC close to 0.90 .

Despite the promising results, diagnostic usage of miRNA biomarkers raises several technical problems. Expression levels of most miRNAs are lower and more variable as compared with mRNAs. Therefore, sensitive quantitative methods are needed for reliable detection of cancer-specific changes of miRNA expression. In our study, miRNA selection for the analysis in urine was performed using TaqMan-based microfluidic plates, which enable precise detection of small deviations in the expression levels of miRNAs in high-throughput settings. However, reliable standardised ECs are indispensable for this relative quantification-based method. RNU6, a small nuclear RNA, is usually included in miRNA TLDA cards by the manufacturer as the most reliable EC for miRNA expression analysis. However, our analysis revealed quite variable expression of this popular EC in prostate tissue. In our TLDA study, six most stably expressed miRNAs in prostate tissue were selected as ECs. However, deregulated expression of some of our selected miRNAs, including let-7g, miR-30c, and miR103, have been reported in PCa (Singh et al, 2014). Owing to this, a second method of data normalisation, that is, global normalisation, was employed in our study and in the studies of other authors (Bandrés et al, 2006). For relative quantification of selected miRNAs in urine specimens, the global normalisation method was also more preferable than the usage of a single selected control miRNA. In addition, the spike-in control (cel-miR-39) was used to eliminate the variation in miRNA isolation efficiency from urine specimens.

Another issue of miRNA-based biomarker studies is the natural variation of the amounts of miRNA in various tissues and biofluids from the same person (Weber et al, 2010). The variation is explained by physiological and pathological conditions, specific miRNA regulation in separate body compartments, and poorly explored ways of miRNA secretion into body fluids. Besides, the levels of urinary miRNAs are highly dependent on urine concentration and sample processing. In our study, miRNAs highly expressed in cancerous prostate tissues were selected for the urine-based assay. However, only miR-375 was abundant in urine from PCa patients, while amounts of other miRNAs were significantly and PCa-specifically reduced. Similar phenomenon was reported in urine from breast cancer patients (Erbes et al, 2015) and explained by specific miRNA secretion into the urinary tract. Importantly, in our study, quite similar levels of urinary miRNAs were detectable in catheterised and voided urine, suggesting an equal utility of differently collected urine specimens for miRNA-based biomarker assay. In urological malignancies, urine is the easily accessible and disease-specific clinical material, thus selective release of particular miRNAs into urinary flow demands further investigations.

\section{CONSLUSIONS}

In our study, several PCa-specific miRNAs were successfully detected in the urine of PCa patients and showed different abundance when compared with urine from ASCs and BPH patients. This reveals suitability of some urinary miRNAs for noninvasive and specific detection of PCa, even at the early stages of the disease. Moreover, our study indicates that combined analysis of urinary miR-375 and miR-148a can significantly improve the specificity and sensitivity of the PSA test, especially in men who fall in the intermediate PSA range of $4-10 \mathrm{ng} \mathrm{ml}^{-1}$. 


\section{ACKNOWLEDGEMENTS}

This research was funded by European Social Fund under National Integrated Programme Biotechnology and Biopharmacy Grant VP1-3.1-ŠMM-08-K-01-005 and supported by COST action CM1406. We thank Vaidotas Stankevicius for participation in performing TLDA experiments.

\section{CONFLICT OF INTEREST}

The authors declare no conflict of interest.

\section{REFERENCES}

Ambs S, Prueitt RL, Yi M, Hudson RS, Howe TM, Petrocca F, Wallace TA, Liu CG, Volinia S, Calin GA, Yfantis HG, Stephens RM, Croce CM (2008) Genomic profiling of microRNA and messenger RNA reveals deregulated microRNA expression in prostate cancer. Cancer Res 68 : 6162-6170.

Bandrés E, Cubedo E, Agirre X, Malumbres R, Zárate R, Ramirez N, Abajo A, Navarro A, Moreno I, Monzó M, García-Foncillas J (2006) Identification by real-time PCR of 13 mature microRNAs differentially expressed in colorectal cancer and non-tumoral tissues. Mol Cancer 5: 29.

Brase JC, Johannes M, Schlomm T, Fälth M, Haese A, Steuber T, Beissbarth T, Kuner R, Sültmann H (2011) Circulating miRNAs are correlated with tumor progression in prostate cancer. Int J Cancer 128: 608-616.

Bray F, Lortet-Tieulent J, Ferlay J, Forman D, Auvinen A (2010) Prostate cancer incidence and mortality trends in 37 European countries: an overview. Eur J Cancer 46: 3040-3052.

Bryant RJ, Pawlowski T, Catto JW, Marsden G, Vessella RL, Rhees B, Kuslich C, Visakorpi T, Hamdy FC (2012) Changes in circulating microRNA levels associated with prostate cancer. Br J Cancer 106: 768-774.

Chen J, Zhang X, Wang Y, Ye Y, Huang Z (2015) Formononetin promotes proliferation that involves a feedback loop of microRNA-375 and estrogen receptor alpha in estrogen receptor-positive cells. Mol Carcinog 55: 312-319.

Cheng HH, Mitchell PS, Kroh EM, Dowell AE, Chéry L, Siddiqui J, Nelson PS, Vessella RL, Knudsen BS, Chinnaiyan AM, Pienta KJ, Morrissey C, Tewari M (2013) Circulating microRNA profiling identifies a subset of metastatic prostate cancer patients with evidence of cancer-associated hypoxia. PLoS One 8: e69239.

Corcoran C, Rani S, O'Driscoll L (2014) miR-34a is an intracellular and exosomal predictive biomarker for response to docetaxel with clinical relevance to prostate cancer progression. Prostate 74: 1320-1334.

Cortez MA, Bueso-Ramos C, Ferdin J, Lopez-Berestein G, Sood AK, Calin GA (2011) MicroRNAs in body fluids-the mix of hormones and biomarkers. Nat Rev Clin Oncol 8: 467-477.

Daniunaite K, Jarmalaite S, Kalinauskaite N, Petroska D, Laurinavicius A, Lazutka JR, Jankevicius F (2014) Prognostic value of RASSF1 promoter methylation in prostate cancer. J Urol 192: 1849-1855.

Demidenko R, Razanauskas D, Daniunaite K, Lazutka JR, Jankevicius F, Jarmalaite $S$ (2015) Frequent down-regulation of $A B C$ transporter genes in prostate cancer. BMC Cancer 15: 683.

Dijkstra S, Mulders PF, Schalken JA (2014) Clinical use of novel urine and blood based prostate cancer biomarkers: a review. Clin Biochem 47: 889-896.

Duffy MJ (2014) PSA in screening for prostate cancer: more good than harm or more harm than good? Adv Clin Chem 66: 1-23.

Epstein JI, Allsbrook WC, Amin MB, Egevad LL. ISUP Grading Committee (2005) The 2005 International Society of Urological Pathology (ISUP) Consensus Conference on Gleason Grading of Prostatic Carcinoma. Am J Surg Pathol 29: 1228-1242.

Erbes T, Hirschfeld M, Rücker G, Jaeger M, Boas J, Iborra S, Mayer S, Gitsch G, Stickeler E (2015) Feasibility of urinary microRNA detection in breast cancer patients and its potential as an innovative non-invasive biomarker. BMC Cancer 15: 193.

Fujita Y, Kojima K, Ohhashi R, Hamada N, Nozawa Y, Kitamoto A, Sato A, Kondo S, Kojima T, Deguchi T, Ito M (2010) MiR-148a attenuates paclitaxel resistance of hormone-refractory, drug-resistant prostate cancer PC3 cells by regulating MSK1 expression. J Biol Chem 285: 19076-19084.

Guzel E, Karatas OF, Semercioz A, Ekici S, Aykan S, Yentur S, Creighton CJ, Ittmann M, Ozen M (2015) Identification of microRNAs differentially expressed in prostatic secretions of patients with prostate cancer. Int $J$ Cancer 136: 875-879.

Haj-Ahmad TA, Abdalla MA, Haj-Ahmad Y (2014) Potential urinary miRNA biomarker candidates for the accurate detection of prostate cancer among benign prostatic hyperplasia patients. J Cancer 5: 182-191.

Huang X, Yuan T, Liang M, Du M, Xia S, Dittmar R, Wang D, See W, Costello BA, Quevedo F, Tan W, Nandy D, Bevan GH, Longenbach S, Sun Z, Lu Y, Wang T, Thibodeau SN, Boardman L, Kohli M, Wang L (2015) Exosomal miR-1290 and miR-375 as prognostic markers in castration-resistant prostate cancer. Eur Urol 67: 33-41.

Jackson BL, Grabowska A, Ratan HL (2014) MicroRNA in prostate cancer: functional importance and potential as circulating biomarkers. $B M C$ Cancer 14: 930.

Jalava SE, Urbanucci A, Latonen L, Waltering KK, Sahu B, Jänne OA, Seppälä J, Lähdesmäki H, Tammela TL, Visakorpi T (2012) Androgenregulated miR-32 targets BTG2 and is overexpressed in castrationresistant prostate cancer. Oncogene 31: 4460-4471.

Kelly BD, Miller N, Healy NA, Walsh K, Kerin MJ (2013) A review of expression profiling of circulating microRNAs in men with prostate cancer. BJU Int 111: 17-21.

Mlcochova H, Hezova R, Stanik M, Slaby O (2014) Urine microRNAs as potential noninvasive biomarkers in urologic cancers. Urol Oncol 32: 41.e1-41.e9.

Murata T, Takayama K, Katayama S, Urano T, Horie-Inoue K, Ikeda K, Takahashi S, Kawazu C, Hasegawa A, Ouchi Y, Homma Y, Hayashizaki Y, Inoue S (2010) miR-148a is an androgen-responsive microRNA that promotes LNCaP prostate cell growth by repressing its target CAND1 expression. Prostate Cancer Prostatic Dis 13: 356-361.

Nguyen HC, Xie W, Yang M, Hsieh CL, Drouin S, Lee GS, Kantoff PW (2013) Expression differences of circulating microRNAs in metastatic castration resistant prostate cancer and low-risk, localized prostate cancer. Prostate 73: $346-354$.

Payne SR, Serth J, Schostak M, Kamradt J, Strauss A, Thelen P, Model F, Day JK, Liebenberg V, Morotti A, Yamamura S, Lograsso J, Sledziewski A, Semjonow A (2009) DNA methylation biomarkers of prostate cancer: confirmation of candidates and evidence urine is the most sensitive body fluid for non-invasive detection. Prostate 69: $1257-1269$.

Roberts MJ, Chow CWK, Schirra HJ, Richards R, Buck M, Selth LA, Doi SA, Samaratunga H, Perry-Keene J, Payton D, Yaxley J, Lavin MF, Gardiner RA (2015) Diagnostic performance of expression of PCA3, Hepsin and miR biomarkers inejaculate in combination with serum PSA for the detection of prostate cancer. Prostate 75: 539-549.

Sabaliauskaite R, Jarmalaite S, Petroska D, Dasevicius D, Laurinavicius A, Jankevicius F, Lazutka JR (2012) Combined analysis of TMPRSS2-ERG and TERT for improved prognosis of biochemical recurrence in prostate cancer. Genes Chromosomes Cancer 51: 781-791.

Sapre N, Selth LA (2013) Circulating microRNAs as biomarkers of prostate cancer: the state of play. Prostate Cancer 2013: ID 539680.

Schaefer A, Jung M, Mollenkopf HJ, Wagner I, Stephan C, Jentzmik F, Miller K, Lein M, Kristiansen G, Jung K (2010) Diagnostic and prognostic implications of microRNA profiling in prostate carcinoma. Int J Cancer 126: $1166-1176$.

Selth LA, Roberts MJ, Chow CWK, Marshall VR, Doi SAR, Vincent AD, Butler LM, Lavin MF, Tilley WD, Gardiner RA (2014) Human seminal fluid as a source of prostate cancer-specific microRNA biomarkers. Endocr Relat Cancer 21: L17-L21.

Selth LA, Townley S, Gillis JL, Ochnik AM, Murti K, Macfarlane RJ, Chi KN, Marshall VR, Tilley WD, Butler LM (2012) Discovery of circulating microRNAs associated with human prostate cancer using a mouse model of disease. Int J Cancer 131: 652-661.

Singh PK, Preus L, Hu Q, Yan L, Long MD, Morrison CD, Nesline M, Johnson CS, Koochekpour S, Kohli M, Liu S, Trump DL, SuchestonCampbell LE, Campbell MJ (2014) Serum microRNA expression patterns that predict early treatment failure in prostate cancer patients. Oncotarget 5: $824-840$.

Sita-Lumsden A, Dart DA, Waxman J, Bevan CL (2013) Circulating microRNAs as potential new biomarkers for prostate cancer. Br J Cancer 108: 1925-1930. 
Szczyrba J, Löprich E, Wach S, Jung V, Unteregger G, Barth S, Grobholz R, Wieland W, Stöhr R, Hartmann A, Wullich B, Grässer F (2010) The microRNA profile of prostate carcinoma obtained by deep sequencing. Mol Cancer Res 8: 529-538.

Taylor DD, Gercel-Taylor C (2008) MicroRNA signatures of tumor-derived exosomes as diagnostic biomarkers of ovarian cancer. Gynecol Oncol 110: $13-21$.

Velonas VM, Woo HH, dos Remedios CG, Assinder SJ (2013) Current status of biomarkers for prostate cancer. Int J Mol Sci 14: 11034-11060.

Wach S, Nolte E, Szczyrba J, Stöhr R, Hartmann A, Ørntoft T, Dyrskjøt L, Eltze E, Wieland W, Keck B, Ekici AB, Grässer F, Wullich B (2012) MicroRNA profiles of prostate carcinoma detected by multiplatform microRNA screening. Int J Cancer 130: 611-621.

Weber JA, Baxter DH, Zhang S, Huang DY, Huang KH, Lee MJ, Galas DJ, Wang K (2010) The microRNA spectrum in 12 body fluids. Clin Chem 56: $1733-1741$.
Xu L, Qi X, Duan S, Xie Y, Ren X, Chen G, Yang X, Han L, Dong Q (2014) MicroRNAs: potential biomarkers for disease diagnosis. Biomed Mater Eng 24: 3917-3925.

Yun SJ, Jeong P, Kang HW, Kim Y-H, Kim E-A, Yan C, Choi YK, Kim D, Kim JM, Kim SK, Kim SY, Kim ST, Kim WT, Lee OJ, Koh GY, Moon SK, Kim IY, Kim J, Choi YH, Kim WJ (2015) Urinary microRNAs of prostate cancer: virus-encoded HSV1-miRH18 and HSV2-miR-H9-5p could be valuable diagnostic markers. Int Neurourol J 19: 74-84.

Ziaran S, Varchulova Novakova Z, Bohmer D, Danisovic L (2015) Biomarkers for determination prostate cancer: implication for diagnosis and prognosis. Neoplasma 62: 683-691.

This work is published under the standard license to publish agreement. After 12 months the work will become freely available and the license terms will switch to a Creative Commons AttributionNonCommercial-Share Alike 4.0 Unported License.

Supplementary Information accompanies this paper on British Journal of Cancer website (http://www.nature.com/bjc) 